\title{
EFFICIENT COLOR IMAGE INDEXING AND RETRIEVAL USING A VECTOR-BASED SCHEME
}

\author{
D. Androutsos \& A.N. Venetsanopoulos \\ Dept. of Elect. \& Comp. Engineering \\ University of Toronto \\ 10 King's College Rd. \\ Toronto, Ontario, M5S 3G4 \\ CANADA \\ \{zeus,anv\}@dsp.toronto.edu
}

\author{
K.N. Plataniotis \\ School of Computer Science \\ Ryerson Polytechnic Univ. \\ 350 Victoria Street \\ Toronto, Ontario, M5B 2K3 \\ CANADA \\ kplatani@acs.ryerson.ca
}

\begin{abstract}
Color is the characteristic which is most used for image indexing and retrieval. Due to its simplicity, the color histogram remains the most commonly used method for color indexing and retrieval. However, the lack of good perceptual histogram similarity measures, the global color content of histograms and the erroneous retrieval results due to gamma nonlinearity, calls for improved methods. We implement a vector angular-based distance measure for image retrieval based on color. We build distance vectors in a multidimensional query space in which the retrieval ranking of each image is determined. Our system exhibits high flexibility by allowing all types of queries, including query by color, query by multiple colors and query by example. In addition, colors can be excluded in a query, without requiring an additional level of analysis.
\end{abstract}

\section{INTRODUCTION}

Content-Based Image Retrieval (CBIR) is a research area dedicated to the image retrieval problem. There are a number of image and video database systems which have recently been developed and others that are currently under development $[1,2]$.

Color remains the most important low-level feature which is used to build indices for database images. Specifically, the color histogram remains the most popular index, due primarily to its simplicity $[3,4]$.

However, using the color histogram for indexing has a number of drawbacks. Specifically, histograms require quantization to reduce dimensionality, color space selection can have a profound effect on the retrieval results and excluding colors in the query is difficult.

In this paper we present a scheme for indexing and retrieving color image data, which addresses the drawbacks with histogram techniques and instead implements vector techniques for indexing and retrieval. We use color segmentation to extract regions of perceptually prominent color and use repre- 
sentative vectors from these extracted regions in the image indices. We end up with a very small index and base similarity on an angular distance measure between a query color vector and the indexed representative vectors.

To build indices into our image database we take into consideration factors such as human color perception and recall. Humans describe the color content of an image, with terms such as red or dark yellow, not RGB values. The color granularity provided by histogram indexing is, in most cases, not necessary, especially when the final observer is a human. Thus, it is more natural to segment an image into regions of similar color and retrieve candidate images based on the similarity to the color of that region.

\section{Segmentation}

Our method of color indexing implements recursive HSV-space segmentation to extract regions within the image which contain perceptually similar color. Hue is particularly important, since it represents color in a manner which is proven to imitate human color recognition and recall. Specifically, in our method, we threshold the hue histogram, which is known to contain most of the color information, while also taking into account saturation and value information.

The first step is to build a hue histogram for all the bright chromatic pixels, which tend to be colors that have value $>75 \%$ and saturation $\geq 20 \%$. Once the pixels which satisfy this criterion are identified, the hue histogram is built and thresholded into $m$ bright colors.

From the remaining image pixels, saturation and value are used to determine which regions of the image are achromatic. Specifically, it has been found, in the literature and experimentally [5], [6] that colors with value $<25 \%$ can be classified as black, i.e., at the bottom of the HSV cone, and that colors with saturation $<20 \%$ and value $>75 \%$ can be classified as white.

All remaining pixels fall in the chromatic region of the HSV cone. However, there may be a wide range of saturation values. We calculate the saturation histogram of all these remaining chromatic pixels. We threshold each saturation peak and calculate the hue histogram for the pixels contained in each given peak. Each resulting hue histogram is then thresholded accordingly,

The result is an accurate low-level representation of the color content in the image using only $n$ color vectors (Figure 1 ), which requires less storage for the indices and can also index spatial information.

\section{Vector approach}

Studies have shown that measures based on the angle of a color vector produce perceptually accurate retrieval results in the RGB domain [7]. Furthermore, angular measures are chromaticity-based, which means that they operate primarily on the orientation of the color vector in the RGB space and therefore are more resistant to intensity changes. 
Figure 1: Typical image and its HUE-segmented image.
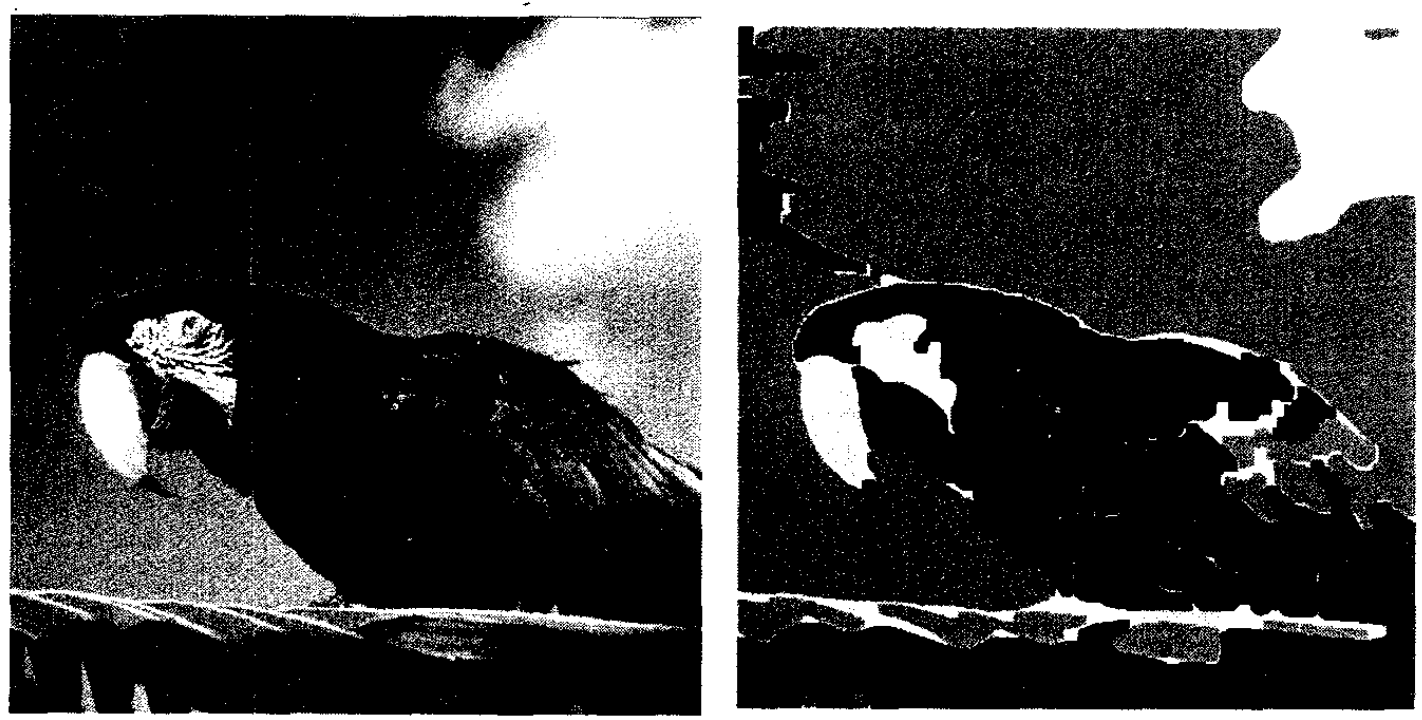

In addition, angular distance measures exhibit excellent performance in the area of image filtering [8]. Retrieval and filtering both use distance measures to determine candidacy. In particular, Order-statistics filters implement distance measures to group similar vectors together and discard outliers, whereas retrieval ranks the similarity between candidates.

In our system we implement a distance measure based on the angular distance between two vectors. Specifically it is a combination distance measure which is composed of an angle and magnitude component:

$$
\delta\left(\vec{x}_{i}, \vec{x}_{j}\right)=1-\underbrace{\left[1-\frac{2}{\pi} \cos ^{-1}\left(\frac{\vec{x}_{i} \cdot \vec{x}_{j}}{\left|\vec{x}_{i}\right|\left|\vec{x}_{j}\right|}\right)\right]}_{\text {angle }} \underbrace{\left[1-\frac{\left|\vec{x}_{i}-\vec{x}_{j}\right|}{\sqrt{3 \cdot 255^{2}}}\right]}_{\text {magnitude }},
$$

where $\vec{x}_{i}$ and $\vec{x}_{j}$ are 3 -dimensional color vectors.

For each query color, the minimum distance between it and the indexed colors is calculated and a multidimensional measure is created which consists of the minimum distances of the query colors to the indexed representative vectors in the given index.

$$
\vec{D}\left(d_{1}, \ldots, d_{n}\right)=\left(\min \left(\delta\left(\vec{q}_{1}, \vec{i}_{1}\right), \ldots, \delta\left(\vec{q}_{1}, \vec{i}_{m}\right)\right), \ldots, \min \left(\delta\left(\vec{q}_{n}, \vec{i}_{1}\right), \ldots, \delta\left(\vec{q}_{n}, \vec{i}_{m}\right)\right)\right)
$$

The database image that is the closest match to the given query colors $q_{1}, q_{2}, \ldots, q_{n}$ is the one which is closest to the origin of the multidimensional distance space. This implies that the distance vector $\vec{D}$ that is most centrally located, i.e, is collinear with the equidistant line of the multidimensional space where all components of $\vec{D}$ are equal and at the same time has the smallest magnitude, corresponds to the image which contains the best match to all the query colors, as shown in Figure 4(a). Figure 2 depicts a user query for at 
least $10 \%$ of the R,G,B colors $26,153,33$ (green) and 200,7,25 (red). Clearly, the displayed top 10 results exhibit colors with strong similarity to the query colors.

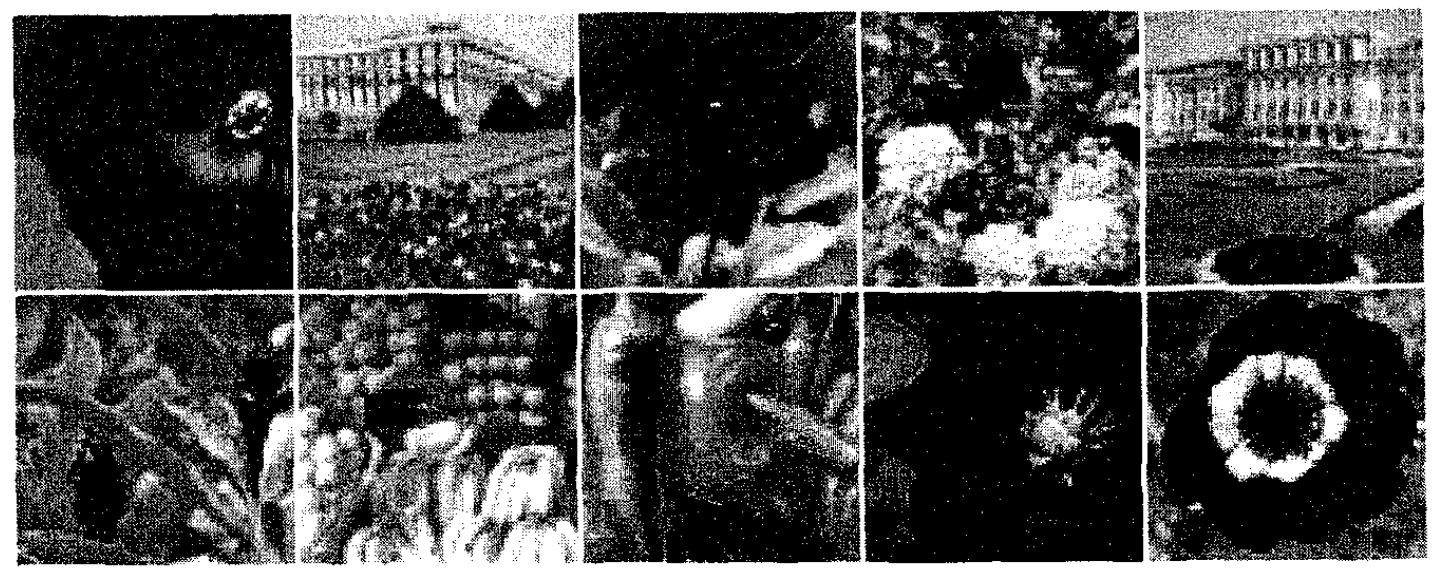

Figure 2: Query result for images with red \& green.

\section{Color exclusion}

Our proposed vector approach provides a framework which easily accepts exclusion in the query process. It allows for image queries containing any number of colors to be excluded in addition to including colors in the retrieval results. From the discussion in Section above, we are interested in distance vectors $\vec{D}$ which are collinear with the equidistant line and which have small magnitude. The exclusion of a certain color should affect $\vec{D}$ accordingly and it's relation to the equidistant line and the origin. For example, if it is found that an image contains an indexed color which is close to an exclusion color, the distance between the two can be used to either pull or push $\vec{D}$ closer or further to the ideal and accordingly affect the retrieval ranking of the given image, as shown in Figure 4(b).

To this end, we determine the minimum distances of each exclusion color with the indexed representative colors, using (2), to quantify how close the indexed colors are to the exclusion colors:

$\vec{X}\left(x_{1}, \ldots, x_{n}\right)=\left(\min \left(\delta\left(\vec{\xi}_{1}, \vec{i}_{1}\right), \ldots, \delta\left(\vec{\xi}_{1}, \vec{i}_{m}\right)\right), \ldots \min \left(\delta\left(\vec{\xi}_{n}, \vec{i}_{1}\right), \ldots, \delta\left(\vec{\xi}_{n}, \vec{i}_{m}\right)\right)\right)$

where $\xi_{n}$ are the $n$ exclusion colors and $i_{m}$ are the $m$ indexed representative colors of each database image. Equation (3) quantifies how similar any indexed colors are to the exclusion colors. To quantify dissimilarity, a transformation of each vector component of $\vec{X}$ is required, and then this is merged with $\vec{D}$ to give the overall multidimensional vector:

$$
\vec{\Delta}=\left[\begin{array}{ll}
\vec{D} & \vec{I}-\vec{X}
\end{array}\right],
$$


where $\vec{I}$ is a vector of size $n$ with all entries of value 1 . The dimensionality of $\vec{\Delta}$ is equal to the number of query colors + number of exclusion colors. The final retrieval rankings are then determined from $|\vec{\Delta}|$ and the angle which $\vec{\Delta}$ in (4) makes with the equidistant line of the query colors. Figure 3 (BOTTOM ROW) depicts the query result when at least $10 \%$ of the R,G,B colors $26,153,33$ (green) and 200, 7, 25 (red) were desired and the color 255, 240,20 (yellow) was excluded. Clearly, images which contained colors closed to yellow were removed from the top ranking results, as compared to the TOP ROW where yellow was not excluded.

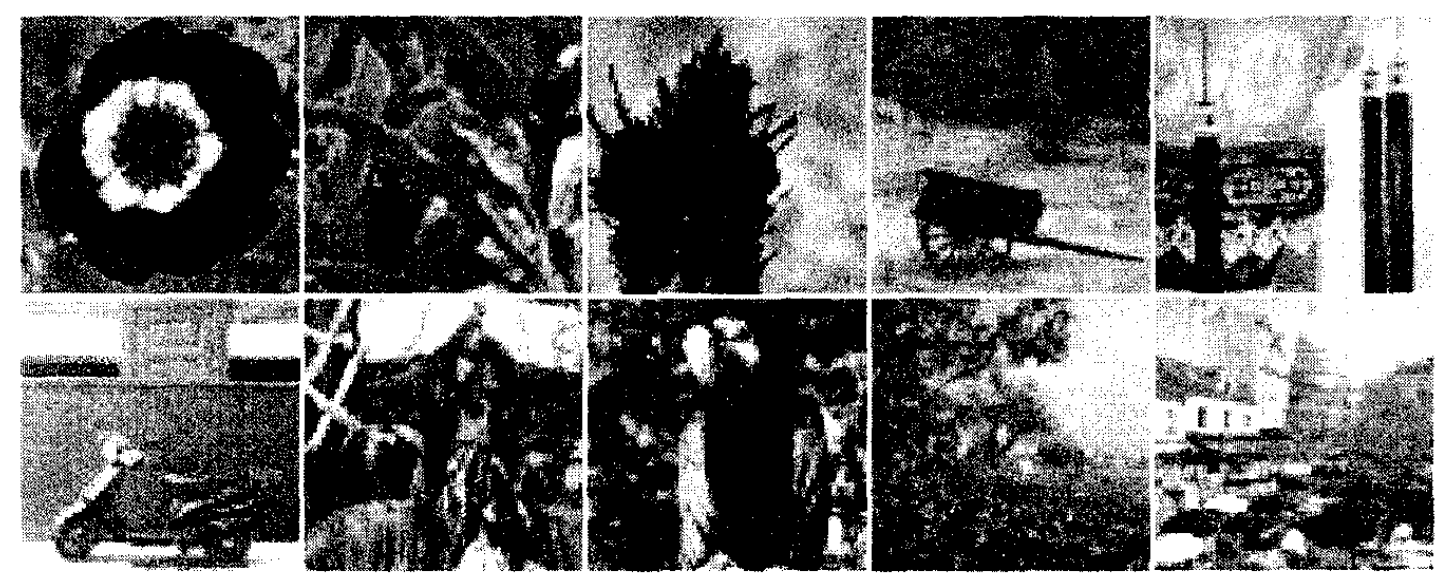

Figure 3: Query result for images with red \& green and excluding yellow .

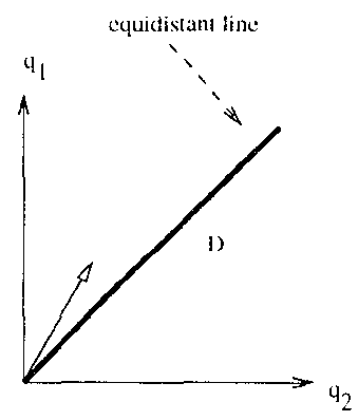

(a)

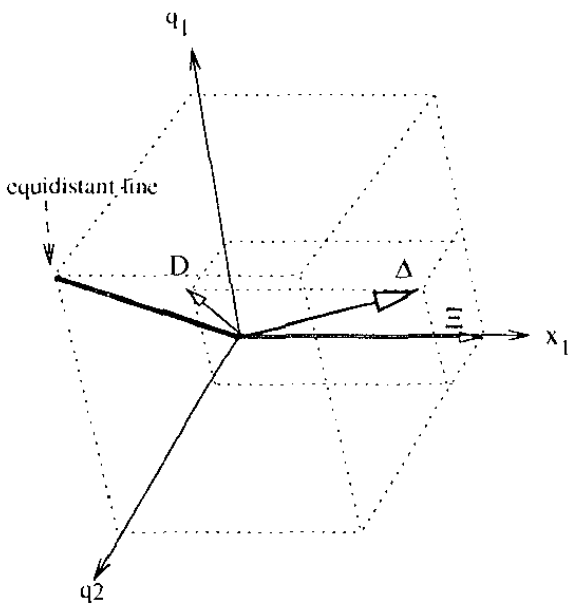

(b)

Figure 4: (a) Vector representation of 2 query colors $q_{1} \& q_{2}$, their multidimensional distance vector $\vec{D}$ and the corresponding equidistant line. (b) the same 2 query colors, 1 exclusion color, $x_{1}$ and the resulting multidimensional distance vector $\vec{\Delta}$. 


\section{CONCLUSIONS}

In this paper we present a new scheme for color image indexing and retrieval. We perform hue segmentation to identify uniform color areas and use the average color vector of these areas as indices into the database. In addition, we also have spatial color information available for indexing. Our system implements a vector angular-based distance measure and a a multidimensional query space which provides great flexibility. Various methods of color query can be performed including color exclusion, where certain colors can be chosen to not appear in the retrieval results.

\section{References}

[1] W. Niblack, R. Barber, W. Equitz, M. Flickner, E. Glasman, D. Petkovic, P. Yanker, C. Faloutsos and G. Taubin, "The QBIC project: Querying images by content using color, texture and shape," Storage and Retrieval for Image and Video Databases, SPIE-1908, San Jose, 1993.

[2] J.R. Smith and S-F. Chang, "VisualSEEK: a fully automated contentbased image query system," ACM Multimedia 96, Boston, November 1996.

[3] X. Wan and C-C. Jay Kuo, "Color distribution analysis and quantization for image retrieval," Storage and Retrieval for Image and Video Databases $I V$, SPIE-2670, pp. 8-16, 1995.

[4] M. Stricker and M. Orengo, "Similarity of color images," Storage and retrieval for image and video databases III, SPIE-2420, pp. 381-392, 1995.

[5] N. Herodotou, K. N. Plataniotis, A. N. Venetsanopoulos, "A contentbased storage and retrieval scheme for image and video databases," $V i$ sual Communications and Image Processing '98, SPIE-3309, San Jose, January, 1998.

[6] Y. Gong, M. Sakauchi, "Detection of regions matching specified chromatic features," Computer Vision and Image Understanding, 61(2), March, 1995.

[7] D. Androutsos, K.N. Plataniotis and A.N. Venetsanopoulos, "Distance Measures for Color Image Retrieval," ICIP '98, Chicago, USA, October 1998.

[8] K.N. Plataniotis, D. Androutsos, S. Vinayagamoorthy, A.N. Venetsanopoulos, "Color Image Processing using Adaptive Multichannel Filters', IEEE Transactions on Image Processing, 6(7), pp. 933-949, September. 1996 